\title{
The Arabidopsis ORGAN SIZE RELATED 2 is involved in regulation of cell expansion during organ growth
}

\author{
Zhixiang Qin ${ }^{1}$, Xiao Zhang ${ }^{1,2}$, Xiaoran Zhang ${ }^{1}$, Guanping Feng ${ }^{3}$ and Yuxin $\mathrm{Hu}^{1,4^{*}}$
}

\begin{abstract}
Background: In plants, the growth of an aerial organ to its characteristic size relies on the coordination of cell proliferation and expansion. These two different processes occur successively during organ development, with a period of overlap. However, the mechanism underlying the cooperative and coordinative regulation of cell proliferation and expansion during organ growth remains poorly understood.

Results: This study characterized a new Arabidopsis ORGAN SIZE RELATED (OSR) gene, OSR2, which participates in the regulation of cell expansion process during organ growth. OSR2 was expressed primarily in tissues or organs undergoing growth by cell expansion, and the ectopic expression of OSR2 resulted in enlarged organs, primarily through enhancement of cell expansion. We further show that OSR2 functions redundantly with ARGOS-LIKE (ARL), another OSR gene that regulates cell expansion in organ growth. Moreover, morphological and cytological analysis of triple and quadruple osr mutants verified that the four OSR members differentially but cooperatively participate in the regulation of cell proliferation and cell expansion and thus the final organ size.

Conclusions: Our results reveal that OSR2 is functional in the regulation of cell expansion during organ growth, which further implicates the involvement of OSR members in the regulation of both cell proliferation and expansion and thus the final organ size. These findings, together with our previous studies, strongly suggest that OSR-mediated organ growth may represent an evolutionary mechanism for the cooperative regulation of cell proliferation and expansion during plant organogenesis.
\end{abstract}

Keywords: Arabidopsis, Cell expansion, Cell proliferation, OSR, Organ size

\section{Background}

For multicellular organisms, organ size is a fundamental attribute of body morphology [1]. In animals, the final size of an organ is primarily determined by cell growth, proliferation, and apoptosis. These processes are primarily mediated by two major pathways: the target of the rapamycin (TOR) pathway that regulates cell growth and the Hippo pathway that coordinately controls cell growth, proliferation, and apoptosis [2,3]. In plants, because cell apoptosis does not generally contribute to morphogenesis in most organs [4], the development of

\footnotetext{
* Correspondence: huyuxin@ibcas.ac.cn

'Key Laboratory of Plant Molecular Physiology, Institute of Botany, Chinese Academy of Sciences, Beijing 100093, China

${ }^{4}$ National Center for Plant Gene Research, Beijing, China

Full list of author information is available at the end of the article
}

an organ to its characteristic size depends mainly on cell proliferation and expansion [5,6]. Recent studies in Arabidopsis have identified a number of genes involved in the regulation of either cell proliferation or cell expansion that are now known to affect final organ size $[7,8]$. However, many such factors appear to be involved in multiple pathways to affect cell number or size [8], suggesting that the mechanisms of organ-size control in plants are more complicated than those in animals [1,9]. This might be partly because plants are immobile and have to be highly responsive to the ever-changing environments [1].

During the development of plant determinate organs such as leaves and floral organs, cell proliferation and expansion occur at two distinct but overlapping phases $[5,6]$. In a leaf, for example, at the early stages of development the cells within leaf primordia undergo a period of cell proliferation and differentiation; cell expansion 
subsequently begins at the leaf tip and proceeds progressively in a basipetal direction, a process during which the transition from cell proliferation to expansion is established [10-12]. Finally, the leaf grows by postmitotic cell expansion and reaches its characteristic final size. Recent studies in Arabidopsis have suggested strongly that the timing of the transition from the cell proliferation to the cell expansion phase appears to be a critical determinant of overall organ size [12-15]. This notion is supported by the large number of identified factors involved in organ-size control that are known to alter the duration of cell proliferation, and thereby alter total cell number and final organ size [6-8]. For instance, some positive regulators that participate in organ-size control such as AINTEGUMENTA (ANT), AUXIN-REGULATED GENE INVOLVED IN ORGAN SIZE (ARGOS), and KLUH/ CYP78A5 (KLU) can prolong the cell proliferation phase and thus increase cell numbers [9,16-18]. There are some negative regulators such as DA1 and ENHANCE OF DA1 (EOD1)/BIG BROTHER (BB), had a role in restricting the period of cell proliferation and thus the organ size [19-21]. Furthermore, factors involved in the regulation of cell expansion during organ growth have been identified in Arabidopsis, including the REGULATORY PARTICLE AAA-ATPASE 2a (RPT2a), EXPANSIN10 (EXP10), ARGOS-LIKE (ARL), and TARGET OF RAPAMYCIN (TOR) [22-26]. These factors were shown to affect either the duration or the rate of cell expansion, and are thus known to alter the final size of cells and of organs. Interestingly, there is a "compensatory mechanism" that coordinates cell proliferation and expansion during organ growth [27-29]. For instance, the an3 mutant had fewer but larger cells than did wild-type plants, and further analysis indicated that the an 3 cells seemed to generate and transmit an intercellular signal that could enhance postmitotic cell expansion [30]. It appears that when the cell number within an organ was decreased below a threshold level, the plants often triggered further post-mitotic cell expansion to compensate for the reduction in cell number $[29,30]$. The mechanisms responsible for the coordination of cell proliferation and expansion during plant organogenesis are far from being understood.

The Arabidopsis ORGAN SIZE RELATED (OSR) family has been implicated in the regulation of aerial organ size $[17,23,31]$. ARGOS, the founding member of the OSR homologues, controlled the growth of aerial organs, mainly through prolonging the duration of cell proliferation by promoting the continued expression of $A N T$ and CycD3;1 [17]. However, ARGOS-LIKE (ARL), a close homolog of $A R G O S$, was found to regulate the organsize by affecting cell expansion [23]. Our recent work demonstrated that ORGAN SIZE RELATED1 (OSR1) promoted organ growth by affecting both cell proliferation and expansion [31]. OSR1, ARGOS, and ARL all share a conserved OSR domain that is sufficient to promote organ growth [31]. Moreover, these three OSR genes are differentially regulated by various plant hormones, suggesting that they may mediate different signals to affect the cell proliferation and/or expansion process. Indeed, a recent study showed that Zea mays ARGOS1 (ZAR1) had a function similar to that of ARGOS. Overexpression of $Z A R 1$ in maize enhanced maize organ growth, increased yield, and improved tolerance to drought stress [32]. OSR genes have only been identified in plants, and it is very interesting that different OSR members with a similar OSR domain could mediate cell proliferation and/or cell expansion, the two different cellular events that have been widely considered to be separately controlled $[9,33,34]$.

There are four OSR homologues in the Arabidopsis genome, and these were likely generated by gene duplication events [31]. To gain further insight into the evolutionary function of the OSR genes, we investigated the role of the fourth OSR gene, OSR2 (At2g41225) during organ growth. Here, we report that OSR2 participates in the regulation of organ growth by primarily affecting cell expansion in a manner redundant with $A R L$. Further analysis of osr 2 argos-1 ARLi triple and argos-1 osr 1 $A R L i$ OSR2i quadruple mutants revealed that these four $O S R$ genes have redundant and cooperative roles in the regulation of cell proliferation and/or expansion. Our characterization of functional divergence among these four $O S R$ genes implies a novel mechanism underlying the coordinative regulation of cell proliferation and expansion during plant organogenesis.

\section{Results}

OSR2 is expressed primarily in organs undergoing growth by cell expansion

We previously reported that Arabidopsis OSR1 was involved in the regulation of organ growth and that it contributes to final organ size primarily through enhancement of cell proliferation [31]. We also showed that three identified OSR proteins shared a conserved OSR domain with an identical LPPLPPPP motif and two putative transmembrane helices, which was sufficient to promote organ growth [31]. The Arabidopsis thaliana genome also has another gene, At2g41225, which encodes a protein of only 67 amino acids that phylogenetically belongs to the OSR member (Additional file 1: Figure S1A) [31]. Careful alignment of its amino acid sequence with those of OSR1, ARGOS, and ARL showed that At2g41225 had the conserved LPPLPPPP motif and the $\mathrm{C}$ terminal transmembrane helix of the OSR domain. However, it lacked the $\mathrm{N}$ terminal transmembrane helix but instead had a plasma membrane-localized signal peptide predicted by Phobius and iPSORT (Additional file 1: Figure S1B) (http://www.ebi.ac.uk//Tools/pfa/phobius; 
http://ipsort.hgc.jp). Moreover, At2g41225 is located alongside OSR 1 in the genome, suggesting that these two genes may originate from a gene duplication event [31]. Therefore, we designated At2g41225 as Organ Size-Related 2 (OSR2).

To explore whether or not OSR2 is functional during organogenesis, we initially monitored OSR2 expression in various organs with qRT-PCR analysis. As shown in Figure 1A, OSR2 was expressed primarily in the expanding organs, including leaf, inflorescence, and flower. We then examined the tissue-specific expression of OSR2 using the T3 independent transgenic plants carrying an OSR2 promoter:ß-glucuronidase (proOSR2:GUS) construct. In two-week-old seedlings, high GUS expression was observed in the organs or organ regions where active cell expansion and elongation were occurring, such as the upper parts of leaves, and the elongating regions of lateral roots (LR); abundant expression was also detected in the cotyledons (Figure 1B-D). However, weak or nearly absent GUS signals were observed

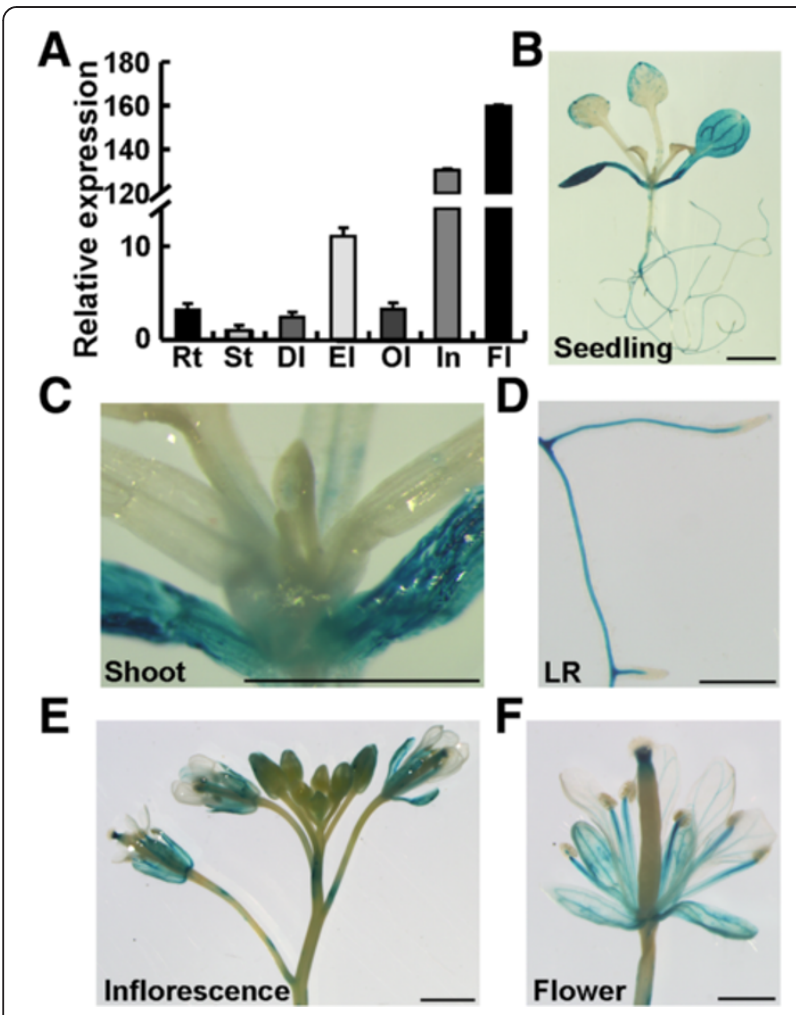

Figure 1 Expression of OSR2. (A) Expression of OSR2 in various organs assayed by qRT-PCR. The data were from three biological replicates and are presented as mean values \pm SE. Rt, root; St, stem; $\mathrm{Dl}$, dividing leaf; El, expanding leaf; Ol, old leaf; In, inflorescence; Fl, flower. (B-F) Expression patterns of OSR2 assayed by GUS staining of transgenic plants carrying a proOSR2:GUS construct. GUS staining was shown in seedling $(\mathbf{B})$, shoot $(\mathbf{C})$, lateral root $(L R, \mathbf{D})$, and the floral organs (E, F). Bars, $2 \mathrm{~mm}$. in juvenile leaves, shoot, and root tips, where cells were predominantly undergoing the cell proliferation process (Figure 1B-D). In the inflorescence and developing floral organs, GUS staining was mainly detected in buds, sepals, and filaments (Figure 1E,F). Careful examination of GUS expression in developing first leaves showed that weak GUS signal was detected at the juvenile stage, while abundant GUS staining was observed when a leaf was undergoing expanding growth. Subsequently, the GUS signal was almost disappeared in fully-expanded leaves (Additional file 1: Figure S1C). These observations indicate that OSR2 is expressed predominantly in organs that are undergoing cell expansion growth.

Since the three previously-identified OSR members were known to be ER-localized [31], we further examined the cellular localization of OSR2 in leaf epidermal cells of proOSR2:OSR2-GFP transgenic plants. Unlike other OSR proteins, the OSR2-GFP fusion protein signal was detected in the plasma membrane (Additional file 1: Figure S1D), indicating that OSR2 is plasma membranelocalized.

\section{Ectopic expression of OSR2 enlarges organs primarily by enhancing cell expansion}

To investigate the role of OSR2 in organ development, we generated transgenic Arabidopsis thaliana (Col-0) plants harboring a pro35S:OSR2 construct. All 35 of the T1 transgenic lines (independent transgenic events) overexpressing OSR2 exhibited, to varying extents, enlarged organs as compared with the empty vector control plants. Careful examination of three of the independent T3 homozygous lines (L1, L2, L3) showed that the ectopic expression of OSR2 resulted in an obviously increased size of various organs such as leaves, cotyledons, roots, floral organs, and siliques (Figure 2A, Additional file 1: Figure S2A, B). Detailed quantification of fully expanded third leaves revealed that the average blade areas of the pro35S:OSR2 L1, L2, and L3 plants were increased by $46.8,44.4$, and $24.1 \%$, respectively, as compared with those of control plants (Figure 2B). qRTPCR analysis verified that the increased sizes of organs were indeed closely associated with the elevated OSR2 expression in these transgenic lines (Figure 2C). The transgenic plants overexpressing OSR2 also displayed longer roots, hypocotyls, siliques, and petioles, and the plant heights were also increased in these plants as compared with the controls (Additional file 1: Figure S2C-F). Additionally, the flowering time in OSR2 transgenic plants was delayed by about four days (Additional file 1: Figure S2E).

We then used leaves and cotyledons as representative organs to investigate the contribution of cell proliferation and cell expansion to the enlarged organs of the p35S: OSR2 plants. As shown in Figure 2D and E, as compared 


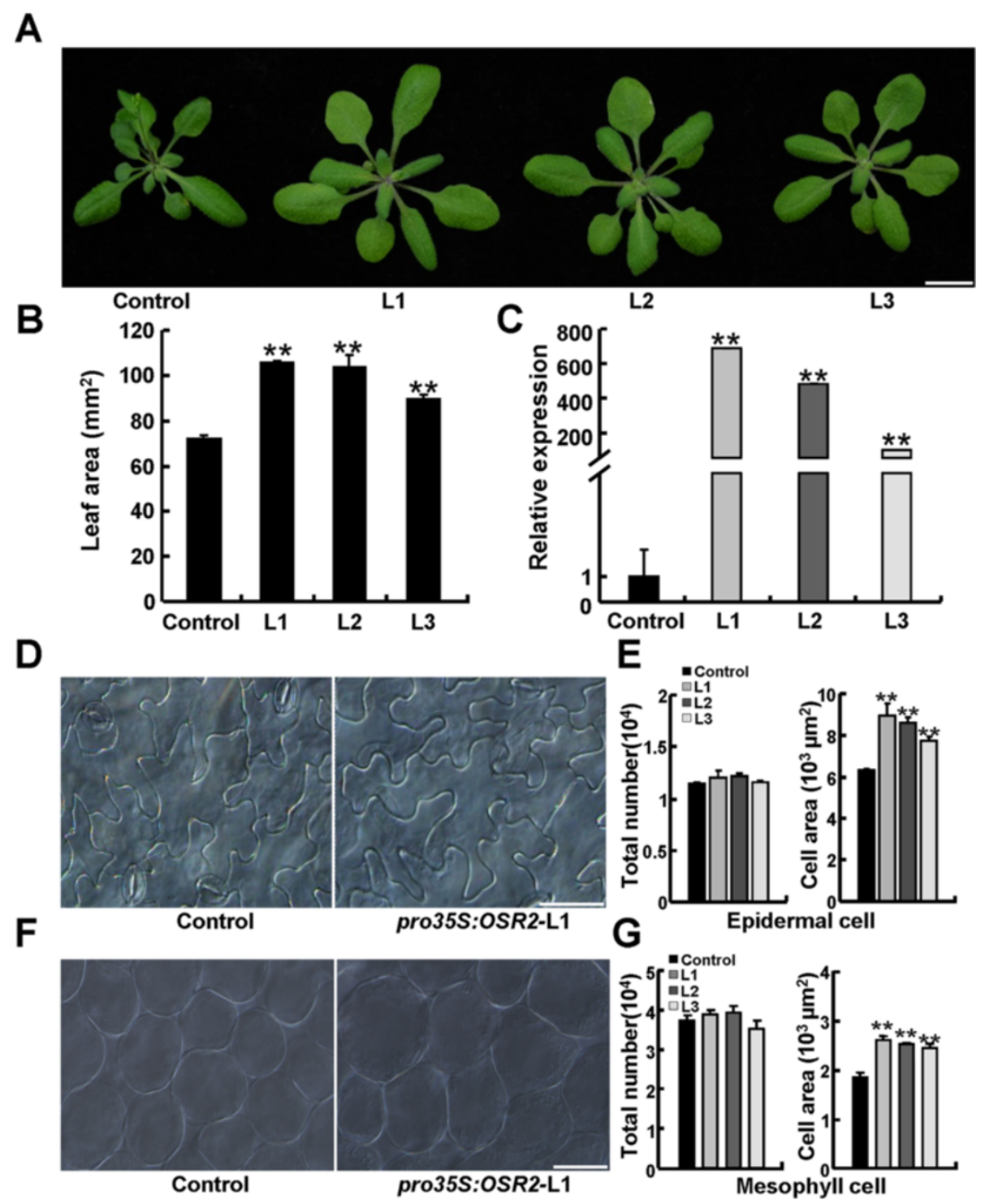

Figure 2 Morphological and cytological characterization of pro35S:OSR2 transgenic plants. (A) Morphology of 28-day-old control and three independent lines of pro35S:OSR2 transgenic plants (L1 to L3). Bar, $1 \mathrm{~cm}$. (B) Blade areas of fully-expanded third leaves of control and three pro35S:OSR2 transgenic lines. At least five leaves for each genotype were examined; data are shown as mean values \pm SE; Student's $t$-test: ${ }^{*} \mathrm{P}<0.01$. (C) qRT-PCR analysis of OSR2 expression in control and transgenic plants presented in (A). The data were from three biological replicates and are presented as mean values \pm SE; Student's $t$-test: ${ }^{* *} P<0.01$. (D, E) The cell area and estimated cell number of epidermal cells in fully-expanded third leaves of control and three pro35S:OSR2 transgenic plants described in (A). At least five leaves were examined for each line, and data are shown as mean values \pm SE; Student's $t$-test: ${ }^{* *} \mathrm{P}<0.01$. Bar, $50 \mu \mathrm{m}$. (F, G) The cell area and estimated cell number of mesophyll cells in fully-expanded third leaves of control and pro35S:OSR2 (three lines) transgenic plants. Bar, $50 \mu \mathrm{m}$. Data are shown as mean values \pm SE; Student's $t$-test: ${ }^{* * P}<0.01$.

with those of the control leaves, the average size of the epidermal cells in fully-expanded third leaves of L1, L2, and L3 plants was increased by $42.3,36.4$, and $22.7 \%$, respectively. The estimated numbers of epidermal cells per leaf were not obviously altered in the transgenic plants (Figure 2E). Similarly, mesophyll cells in p35S:OSR2 leaves were enlarged, whereas the estimated cell numbers did not differ from the control leaves (Figure 2F,G). Consistently, ectopic expression of OSR2 resulted in larger mesophyll cells in cotyledons, and again the mesophyll cell number in cotyledons did not differ from the control (Additional file 1: Figure S3A-C). These observations demonstrate that the enlarged organs in p35S:OSR2 resulted primarily from enhanced cell expansion rather than cell proliferation.

OSR2 affects the cell expansion rate during organ growth To define the exact role of OSR2 in cell expansion rate and duration, we further compared the expansion kinetics 
of epidermal cells in both the tips and the basal regions of the third leaves in control and p35S:OSR2 L1 plants. As shown in Figure $3 \mathrm{~A}$ and $\mathrm{B}$, the cell expansion rates of both the tips and the bases of p35S:OSR2 leaves were indeed much higher than those of controls from day 9 to day 16, whereas the cell expansion rates in the two genotypes were almost the same after day 21; cell expansion ceased after day 27(Figure 3A,B). These

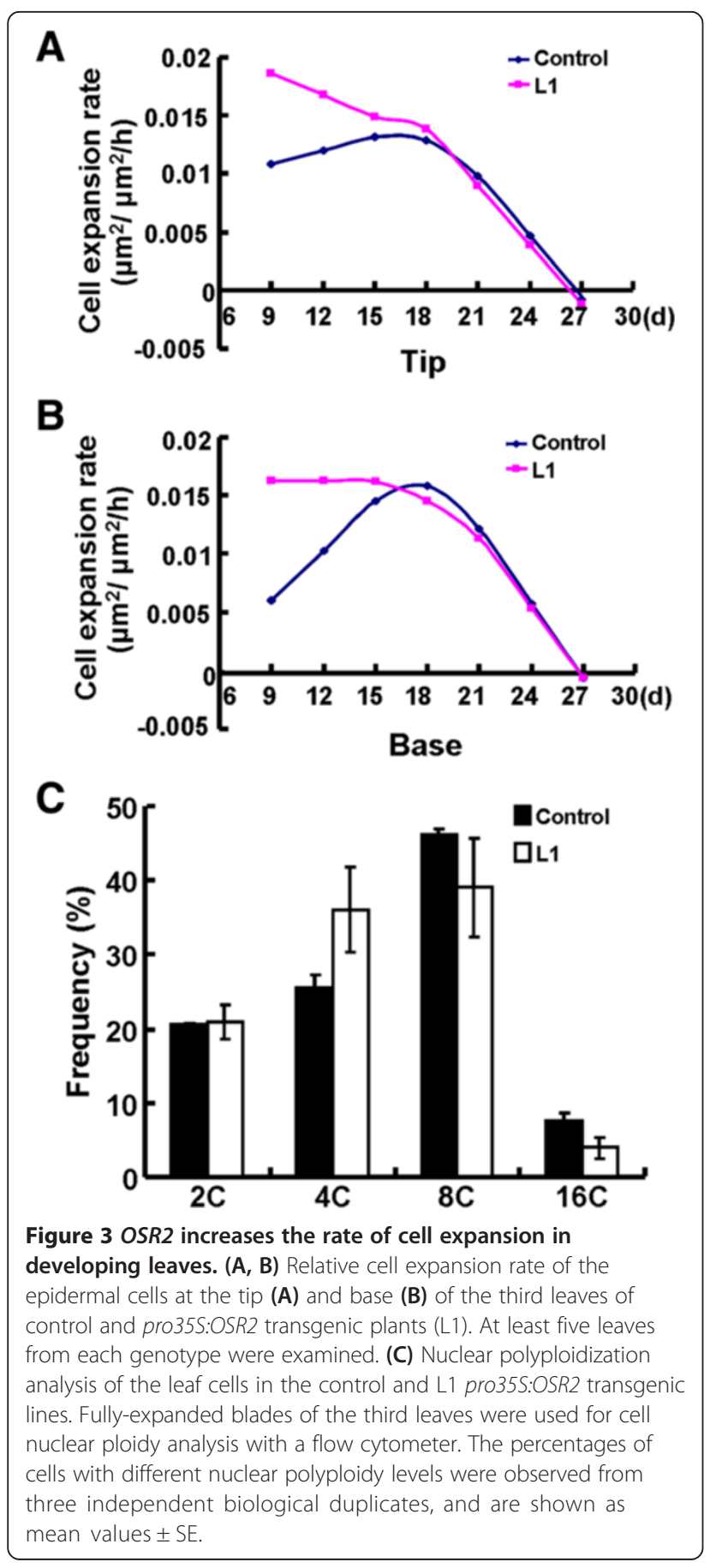

observations strongly suggest that ectopic expression of OSR2 mainly affects the rate of cell expansion rather than the duration of cell expansion phase.

As cell endoreduplication is known to contribute to final cell size $[35,36]$, we investigated whether the enlarged cells in the p35S:OSR2 leaves were attributable to alteration of endoreduplication. Flow cytometry analysis of the nuclei of fully expanded leaves of 27-day-old plants showed that, although the percentages of cells from $4 \mathrm{C}$ and $8 \mathrm{C}$ were slightly different between the two genotypes, the overall distribution of cells from $2 \mathrm{C}$ to $16 \mathrm{C}$ remained comparable between two genotypes (Figure $3 \mathrm{C}$ ). This result indicates that OSR2-mediated cell expansion is not related to the nuclear DNA endoreduplication of leaf cells.

\section{OSR2 functions redundantly with $A R L$ to mediate cell expansion}

To further investigate the role of OSR2 in organ growth, we obtained the T-DNA insertion mutant osr2 (Salk_142851, Col-0) from the Arabidopsis Biological Resource Center (ABRC), in which a T-DNA fragment was inserted into the exon of OSR2 (Additional file 1: Figure S4A). RT-PCR analysis showed that osr 2 was an OSR2 knock-out mutant (Additional file 1: Figure S4B). However, osr2 plants didn't show an obvious reduction in organ size when compared with wild-type (WT) plants (Figure 4A). Since the tissue-specific expression pattern of OSR2 was quite similar to that of $A R L$ (another OSR member that was reported to regulate organ growth by cell expansion [23]), we speculated that OSR2 may have functional redundancy with $A R L$. To test this, we introduced an $A R L$ specific RNA-interference construct (pro35S: ARL RNAi) into both WT and osr 2 plants to generate $A R L i$ and osr 2 $A R L i$ plants. qRT-PCR analysis validated that the expression of $A R L$ was obviously knocked down in the independent T3 lines (Additional file 1: Figure S4C, D). The final size of the third leaf of the $A R L i$ transgenic plants was reduced by about $9 \%$ as compared with that of either the WT or osr 2 . By contrast, leaf size in osr $2 A R L i$ plants L6 and L8 was decreased by about 24.4 and $22.7 \%$, respectively (Figure 4B). Consistently, careful examination of the number and size of epidermal cells in the third leaf confirmed that the knockout of OSR2 led to size reduction of epidermal cells in ARLi plants but had no obvious effect on cell number (Figure 4C,D). Consistently, transgenic plants harboring a $35 \mathrm{~S}$ promoter-driven specific OSR2 RNAi construct (pro35S:OSR2 RNAi) did not exhibit any organ-size phenotype. However, introduction of a pro35S:OSR2 RNAi construct into ARLi plants enhanced the size reduction of leaves (Additional file 1: Figure S4E and F), confirming that OSR2 acts redundantly with $A R L$ in the regulation of cell expansion during organ growth. 


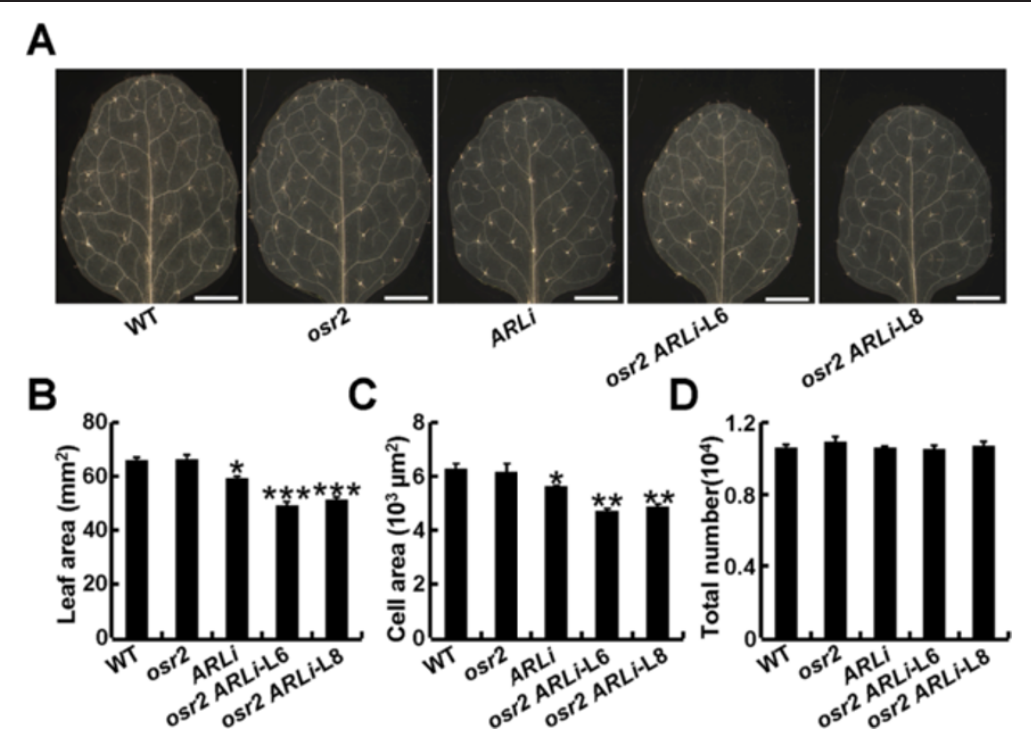

Figure 4 OSR2 acts redundantly with ARL. (A, B) Morphology and the size of third leave of 26-day-old WT, osr2, ARLi, and osr2 ARLi (L6 and L8) plants. At least five fully-expanded leaves of each genotype were used for determination of the leaf size; the data are shown as mean values \pm SE; Student's $t$-test: ${ }^{*} P<0.05$, ${ }^{* * *} P<0.001$. Bars, 2 mm. (C, D) The epidermal cell area and estimated cell number of the third leave described in (A). Data were from at least five leaves of each genotype and are shown as mean values \pm SE; Student's $t$-test: ${ }^{*} P<0.05,{ }^{* * P}<0.01$.

As the three previously-identified OSR genes are known to be differentially responsive to various hormones $[17,23,31]$, we also investigated the transcriptional regulation of OSR2 by plant hormones. Interestingly, the qRTPCR analysis of seedlings treated with various hormones revealed that, similar to $A R L$, OSR2 was induced by epibrassinolide (epi-BL) (Additional file 1: Figure S5A), and this induction was disrupted in the BR signaling mutants bri1-6 and bin2 (Additional file 1: Figure S5B). Moreover, introduction of a p35S:OSR2 construct into bri1-6 and bin 2 could partially restore leaf and petiole growth, and the expansion defect in bri1- 6 and bin2 leaf cells was partially restored by overexpression of OSR2 (Additional file 1: Figure S5C-F). These findings suggest that OSR2 may also be involved in BR-related cell expansion, further supporting the likely functional redundancy between OSR2 and $A R L$ during organ growth.

\section{OSR genes cooperatively regulate cell proliferation and expansion during organogenesis}

Our previous work revealed that ARGOS and OSR1 regulated organ growth by primarily affecting cell proliferation in a redundant manner [31]. To examine the cooperative role of the OSR family members in organ growth, we generated an osr 2 argos- 1 ARLi triple mutant and an argos-1 osr1 ARLi OSR2i quadruple mutant by introducing a pro35S:OSR2 RNAi construct into argos-1 osr1 ARLi plants (Figure 5A). qRT-PCR analysis confirmed that the expression of $A R L$ was knocked down in the osr2 argos-1 ARLi triple mutant, and the expression of both $A R L$ and OSR2 transcripts was decreased in the argos-1 osr1 ARLi OSR2i quadruple mutant (Additional file 1: Figure S6A, B). As expected, the introduction of argos mutation to osr 2 ARLi plants further reduced the final size of their organs, and this reduction was enhanced in the quadruple mutant argos-1 osr1 ARLi OSR2i plants (Figure 5A,B). Cytological analysis of epidermal cells of the third leaves in these plants indicated that this reduction was primarily caused by a decrease in cell numbers (Figure 5C,D), confirming the role of $A R G O S$ and OSR1 in cell proliferation. These differential roles of $O S R$ genes in cell proliferation or expansion were further verified by examining the kinetics of cell proliferation and expansion during organ growth in these genotypes (Figure 5E,F). Notably, we observed that epidermal cells of leaves in the OSR quadruple mutant were slightly smaller than those of the osr $2 A R L i$ plants (Figure 5C,E). This is consistent with the previous finding that OSR1 had some effects on cell expansion during organogenesis [31].

\section{Discussion}

Characterization of OSR2 defines a new OSR member involved in the regulation of organ growth

The development of an organ relies on the coordination of cell proliferation and expansion; these two different cellular events determine the overall organ size. Cell proliferation, which sets the cell number of an organ, plays an important role in organ growth and final size control [8]. Post-mitotic cell expansion, which determines 


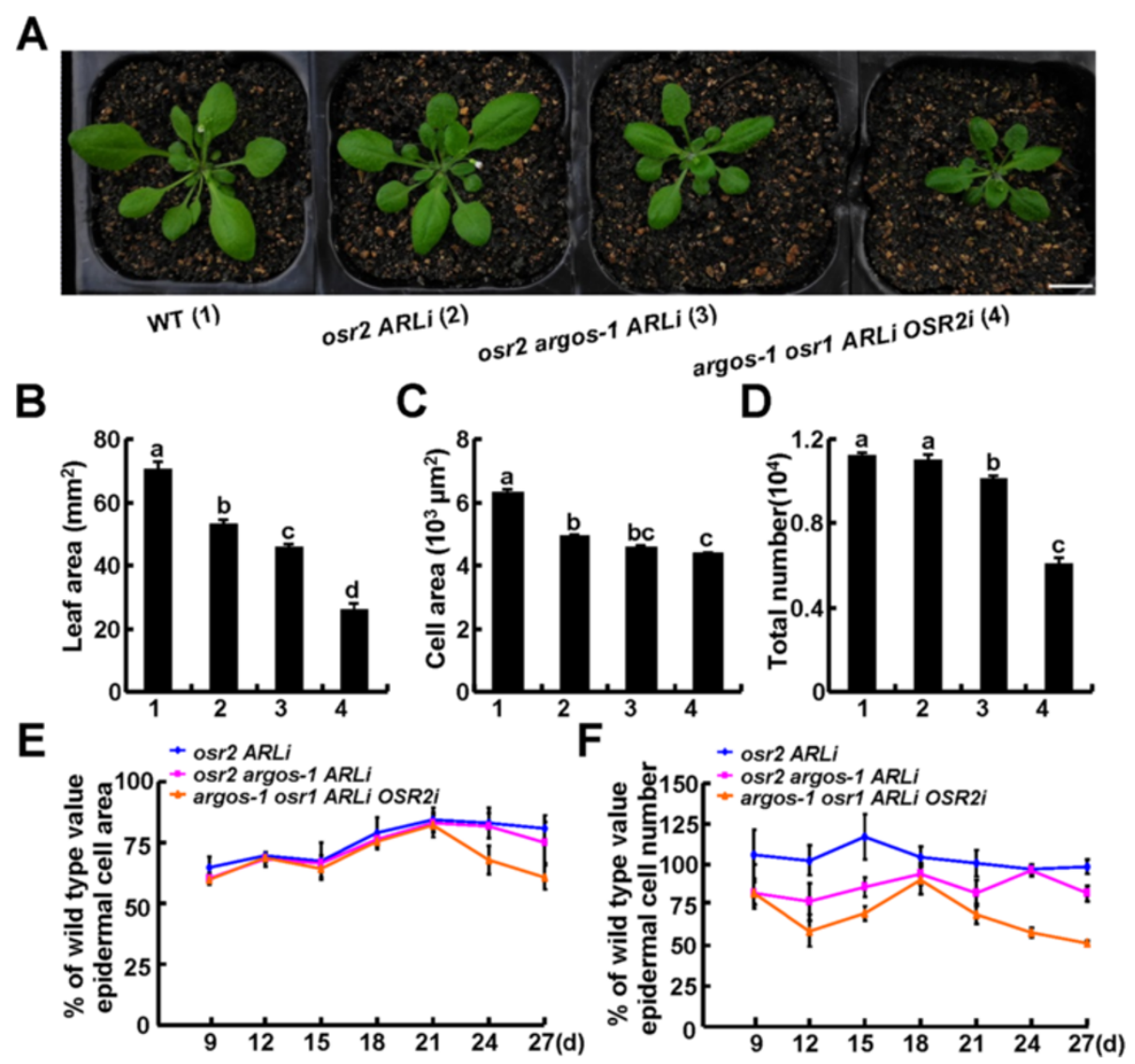

Figure 5 Cooperative roles of OSR2 and other OSR genes in cell proliferation and expansion. (A) Morphology of 28-day-old WT, oSr2 ARLi, oSr2 argos-1 ARLi, and argos-1 osr1 ARLi OSR2i plants. Bar, 1 cm. (B-D) The leaf area (B), epidermal cell area (C), and estimated epidermal cell number (D) of the third leaves of the 30-day-old plants described in (A). At least five leaves were examined for each genotype; the data are shown as mean values $\pm \mathrm{SE}$; the letters (a to d) indicate statistical significance $(P<0.05)$ among the genotypes according to one-way ANOVA testing (SPSS 13.0, Chicago, IL, USA). (E, F) The cellular kinetics of developing third leaves of WT and osr mutant plants. The epidermal cell size (E) and estimated epidermal cell number (F) are shown as the ratio of mutant/WT from 9-27 day-old plants. At least five leaves of each genotype were examined.

the final cell size, is also critical for organ development and size regulation [37]. Recently, several genes that participate in the regulation of cell expansion and thus cell size have been also reported to affect final organ size, such as EXP10, RPT2a, TOR, ARL, and ErbB-3 EPIDERMAL GROWTH FACTOR RECEPTOR BINDING PROTEIN 1 (EBP1) [8]. Here, we showed that OSR2 participates in the regulation of cell expansion during organ growth. OSR2 was expressed in the organs or tissues undergoing cell expansion and elongation, and ectopic expression of OSR2 increased overall organ size by enhancing the cell expansion rate. Although the lossof-function mutant of OSR2 had no obvious phenotype, our detailed examination revealed that OSR2 acted redundantly with $A R L$, another OSR member that had been shown previously to affect cell expansion and thus final organ size [23]. Consistently, the expression pattern of OSR2 in various organs and the response of OSR2 expression to hormone treatment were quite similar to those of $A R L$, supporting the supposition that these genes have functional redundancy in organ growth. Therefore, our work identified OSR2 as a new regulator of plant organ growth and final organ size, thereby providing an alternative to use OSR2 in efforts to manipulate biomass production in plants.

OSR-mediated organ growth may represent an evolutionary mechanism of the cooperative regulation of cell proliferation and expansion

There are four OSR genes in the Arabidopsis genome, and phylogenetic analysis showed that these four genes likely originated from gene duplication events. ARGOS and $A R L$ were clustered into a group and OSR1 and OSR2 were clustered into a separate group [31]. OSR1 and OSR2 are located adjacent in the Arabidopsis genome. Surprisingly, ARGOS and OSR1 are expressed predominately in 
organs or tissues undergoing growth by cell proliferation, and they are known to act upstream of $A N T$ and redundantly regulate the duration of cell proliferation and thus the final organ size [17,31]. OSR2 and ARL are expressed primarily in organs and tissues in which cell expansion/ elongation is occurring, and our work here demonstrates that these genes mediate the cell expansion process and thus organ size. It is interesting that the different members of a co-evolved family function differentially in the regulation of cell proliferation or expansion, two fundamental cellular events that have long been considered to be separately controlled in multicellular organisms $[9,33,34]$. Therefore, OSR-mediated organ growth might represent an evolved mechanism of cooperative control of cell division and expansion at the organ level. Since OSRs were identified only in plants, and OSR mutations only resulted in organ growth phenotypes, it is likely that the OSR regulatory pathway represents a mechanism with which plants respond to growth signals or environmental cues to modify their growth and final organ size. Indeed, the $O S R$ genes were transcriptionally regulated by treatment with different hormones. ARGOS expression is known to be induced by auxin and cytokinin; OSR1 expression is induced by ethylene, but repressed by $A B A$ and BR; the expression of $A R L$ and OSR2 is induced by BR [17,23,31]; Zea mays ARGOS1 (ZAR1) is known to regulate the final size of maize organs and thus contributes to increased yields, particularly in drought stress conditions [32].

\section{The molecular roles of OSR family members during plant organogenesis}

Our characterization of OSR members raises a critical question: how do the different members of the OSR family participate in the regulation of two different cellular events? More importantly, although cell division and cell expansion are strictly coordinated at both the cellular and organ levels, the two processes are generally considered to be controlled by the different mechanisms $[9,33,34]$. There has been little evidence so far that these two events share the same molecular machinery. Moreover, our previous work demonstrated that the OSR domain was sufficient to promote the organ growth, and that OSR1 also had some effect on the cell expansion process [31]. Obviously, the involvement of different OSR genes in cell proliferation and/or expansion relies on their temporal-spatial expression in developing organs; ARGOS and OSR1 are expressed primarily in the cell proliferation phase while $A R L$ and $O S R 2$ are expressed primarily in the cell expansion phase [17,23,31]. It still remains unclear how they facilitate the regulatory role in cell division or expansion with a similar OSR domain. Our previous work showed that three OSR proteins were likely localized to the ER, and here we showed that OSR2 is localized to plasma membrane. We still do not know whether the ER-localized OSR proteins or the plasma membrane-localized OSR2 are functional or processing forms. Because OSRs are small proteins, we also cannot exclude the possibility that OSRs may be functional as peptide signals. Finally, it is more likely that different OSR members may differentially interact with the variety of proteins, which would in turn diverge the roles of OSR in cell proliferation or expansion. Therefore, it will be critical to identify proteins that interact with OSR proteins and to dissect and characterize their downstream targets. Such efforts will be necessary for elucidating the molecular mechanism underlying OSR-mediated cell proliferation and expansion at the organ level.

\section{Conclusions}

In conclusion, we revealed that the Arabidopsis OSR2 participates in regulation of cell expansion during organogenesis and thus the organ size. Our findings of the differential and cooperative roles of OSR genes in regulation of cell proliferation or expansion strongly suggest that OSR-mediated organ growth may represent an evolutionary mechanism of the cooperative regulation of cell proliferation and expansion during plant organogenesis.

\section{Methods}

Plant materials and growth conditions

Arabidopsis thaliana ecotype Columbia (Col-0) was used in this study. osr2 (Salk_142851, Col-0) and argos-1 (SAIL_896_G10, Col-0) were obtained from the Arabidopsis Biological Resource Center (ABRC), and osr1 (GABI_436G04) was from the Nottingham Arabidopsis Stock Centre (NASC) (http://www.arabidopsis.org). All seeds were sterilized in $0.5 \%$ sodium hypochlorite for 15 minutes, and geminated on 1/2 Murashige and Skoog (MS) medium in a culture room at $22^{\circ} \mathrm{C}$ under a $16-\mathrm{h}$ light/8-h dark photoperiod with an illumination intensity of $80-90 \mu \mathrm{mol} \mathrm{m} \mathrm{m}^{-2} \mathrm{~s}^{-1}$. Seven-day-old seedlings were transferred to soil and grown in a growth room at $22 \pm$ $1^{\circ} \mathrm{C}$, under the same photoperiod and illumination regime as those in the culture room [38].

\section{Sequence alignment}

The full length amino acid sequences of OSR2, OSR1, ARGOS, and ARL were obtained from The Arabidopsis Information Resource (TAIR) database. Alignment analysis of the four proteins was performed with the MUSCLE program (http://www.ebi.ac.uk/Tools/msa/muscle/), and manually optimized with Genedoc software [39,40]. The cluster of four proteins was analyzed with CLUSTALW2 (http://www.ebi.ac.uk/Tools/msa/clustalw2). 


\section{Plasmid construction and Arabidopsis transformation}

To generate the pro35S:OSR2 and proOSR2:OSR2-GFP constructs, the coding sequence of OSR2 was amplified by RT-PCR and ligated into the pEASY-Blunt vector (TransGen Biotech, China) and sequenced. The OSR2 fragment digested with appropriate restriction endonuclease(s) was cloned into the pVIP96 or pMDC83 plasmid [17,41]. For the proOSR2:GUS and proOSR2: OSR2-GFP construct, a 1880-bp genomic fragment from the OSR2 promoter was cloned into the pMDC163 vector and the pMDC83 plasmid containing the coding sequence of OSR2 [41]. For the RNAi constructs, a 142-bp cDNA fragment specific for $A R L$ or a 193-bp cDNA fragment specific for OSR2 was cloned into the pBluescript SK plasmid containing an RNAi fragment in both the sense and the antisense orientations [42], and then cloned into pVIP96 or pMDC83 to generate pro35S:ARL RNAi or pro35S:OSR2 RNAi constructs, respectively. All primers used in the generation of these constructs are detailed in Additional file 1: Table S1.

All generated constructs were introduced into Arabidopsis thaliana ecotype Columbia (Col-0) by Agrobacterium tumefaciens-mediated transformation via the described floral dip method [43]. For each construct, a minimum of 18 independent lines harboring a single T-DNA insertion were generated, and three independent lines of their T3 generation plants were used for detailed analysis.

\section{Hormone treatment and gene expression analysis}

For the hormone treatment, nine-day-old seedlings were transferred to $1 / 2 \mathrm{MS}$ liquid medium containing $5 \mu \mathrm{m}$ IAA, $5 \mu \mathrm{m}$ kinetin (KT), $100 \mu \mathrm{m}$ GA3, $5 \mu \mathrm{m}$ ACC, $50 \mu \mathrm{m}$ ABA or $1 \mu \mathrm{m}$ 24-epi-brassinolide (epi-BL) for $3 \mathrm{~h}$. Total RNA from different materials was isolated using TRIzol reagent (Invitrogen). After digestion with DNaseI, RNA was reverse transcribed with Superscript-III reverse transcriptase (Invitrogen) into cDNA for subsequent RT-PCR or qRT-PCR analysis. The transcript abundance of GLYCERALDEHYDE-3-PHOSPHATE DEHYDROGENASE C SUBUNIT (GAPC) or ACTIN2 was used as an internal control in the RT-PCR or qRT-PCR analysis, respectively. The qRT-PCR analysis was performed with SYBR Premix Ex Taq Mix on a Rotor-Gene3000 instrument (Corbett Research) with three biological replicates, according to the manufacturer's instructions. The primers used for expression analyses are detailed in Additional file 1: Table S1.

Seedlings or individual organs of homozygous transgenic plants carrying a proOSR2:GUS construct were used for the GUS staining assay. These were incubated in a $50 \mathrm{mM}$ sodium phosphate solution ( $\mathrm{pH7.0)}$ containing $5 \mathrm{mM} \mathrm{K} 4 \mathrm{Fe}(\mathrm{CN}) 6,5 \mathrm{mM} \mathrm{K3Fe}(\mathrm{CN}) 6,0.1 \%$ Triton $\mathrm{X}-100$, and $1 \mathrm{mM} \mathrm{5-bromo-4-chloro-3-indolyl-b-glucur-}$ onic acid (Gluc) at $37^{\circ} \mathrm{C}$ for several hours [31].

\section{Morphological and cytological analyses}

For morphological characterization, six-day-old etiolated seelings grown in the dark were used for the measurement of the hypocotyl length; eight-day-old seedlings were used for the measurement of cotyledon area and root length; 28-day-old plants were used for the measurement of petiole length; 50-day-old plants were used to determine plant height and silique length.

To determine cell size and number, expanding or fullyexpanded third leaves and cotyledon were excised and placed in a destaining solution for 30 minutes or overnight at room temperature. Then the destaining solution was exchanged with basic solution for 15-20 minutes at room temperature. The materials were rehydrated via an ethanol series for 10-15 minutes and were then transferred in water to visualize and measure cells [44]. The mesophyll and epidermal cells at the tip, central, and basal positions of a leaf were visualized under a microscope and photographed (Olympus BX51), and the areas of leaves and cells were measured with Image 1.4.3.67 software (http://rsb.info.nih.gov/ij/). The total cell number per leaf was estimated as the total leaf area multiplied by the average cell number per area. To determine the cell expansion rate, the measured size of epidermal cells in leaves at different stages were transferred into $\log _{2}$ values and then fitted with a local five point quadratic function, the first derivative of which was calculated according to the method described by Nelissen et al. [45].

\section{Flow cytometric assay and confocal microscopy}

Fully-expanded third leaves of control and transgenic plants overexpressing OSR2 at 27 Day After Germination (DAG) were excised with a razor and then suspended in cold nuclear isolation buffer [46]. Flow cytometric analysis was carried out as described previously with a FACS Caliber flow cytometer (BD Biosciences, http://www. bdbiosciences.com/).

To examine the cellular localization of OSR2, the epidermal cells of transgenic plants harboring a proOSR2: OSR2-GFP construct were used to visualize GFP signals under a confocal microscope (Leica). FM4-64 staining was used to confirm the plasma membrane localization of the OSR2-GFP fusion protein.

\section{Accession numbers}

The sequence data for the genes mentioned in this work can be found in Arabidopsis Information Resource (TAIR) database as: OSR2 (At2g41225), OSR1 (At2g41230), ARL (At2g44080), ARGOS (At3g59900), GAPC (At3g04120) and ACTIN2 (At3g18780).

\section{Availability of supporting data}

The data supporting the results of this article are included within the article and its additional file. 


\section{Additional file}

Additional file 1: Figure S1. Cluster and alignment of OSR2 and OSR proteins. Figure S2. Phenotypes of p35S:OSR2 transgenic plants. Figure S3. Cytological characterization of cotyledons in p35S:OSR2 transgenic plants. Figure S4. Characterization of osr2 and osr mutants. Figure S5. OSR2 is involved in BR-mediated cell expansion. Figure S6. Molecular characterization of osr mutants. Table S1. The primers used in this study.

\section{Abbreviations}

ABRC: The Arabidopsis biological resource center; DAG: Day after germination; epi-BL: Epi-brassinolide; GUS: $\beta$-glucuronidase; KT: Kinetin; LR: Lateral roots; MS: Murashige and Skoog; OSR: ORGAN SIZE RELATED; RNAi: RNA-interference; TAIR: The Arabidopsis information resource; TOR: The target of the rapamycin; WT: Wild-type.

\section{Competing interests}

The authors declare that they have no competing interests.

\section{Authors' contributions}

$\mathrm{ZQ}$ and $\mathrm{YH}$ designed the experiments and contributed to the manuscript writing; ZQ performed the most of experiments and assays; XZ and GF generated the constructs and transgenic lines; XRZ helped to analyze some data; YH supervised and coordinated all experiments. All authors read and approved the manuscript.

\section{Acknowledgements}

We are grateful to Drs. Zhi-Yong Wang (Carnegie Institution for Science, Stanford University) and Shengwei Zhu (Institute of Botany, Chinese Academy of Sciences) for kindly providing the bri1-6 and bin2-/+ seeds. We also thank Dr. Jingbo Jin (Institute of Botany, Chinese Academy of Sciences) for technical assistance with the cellular localization of OSR2, and Drs. Jie Le and Junjie Zou (Institute of Botany, Chinese Academy of Sciences) for help with the cytological analysis. This work was supported by grants from the National Natural Science Foundation of China (31121065 and 31260066).

\section{Author details}

'Key Laboratory of Plant Molecular Physiology, Institute of Botany, Chinese Academy of Sciences, Beijing 100093, China. ${ }^{2}$ University of Chinese Academy of Sciences, Beijing, China. ${ }^{3}$ Key Laboratory for Biodiversity Science and Ecological Engineering, School of Life Sciences, Jinggangshan University, Ji'an, Jiangxi 343009, China. ${ }^{4}$ National Center for Plant Gene Research, Beijing, China.

Received: 25 July 2014 Accepted: 25 November 2014

Published online: 10 December 2014

\section{References}

1. Horiguchi G, Ferjani A, Fujikura U, Tsukaya H: Coordination of cell proliferation and cell expansion in the control of leaf size in Arabidopsis thaliana. J plant Res 2006, 119(1):37-42.

2. Pan D: Hippo signaling in organ size control. Genes Dev 2007, 21(8):886-897.

3. Pan D, Dong J, Zhang Y, Gao XS: Tuberous sclerosis complex: from Drosophila to human disease. Trends Cell Biol 2004, 14(2):78-85.

4. Krizek BA: Making bigger plants: key regulators of final organ size. Curr Opin Plant Biol 2009, 12(1):17-22.

5. Mizukami Y: A matter of size: developmental control of organ size in plants. Curr Opin Plant Biol 2001, 4(6):533-539.

6. Anastasiou E, Lenhard M: Growing up to one's standard. Curr Opin Plant Biol 2007, 10(1):63-69.

7. Gonzalez N, Beemster GT, Inzé D: David and Goliath: what can the tiny weed Arabidopsis teach us to improve biomass production in crops? Curr Opin Plant Biol 2009, 12(2):157-164.

8. Gonzalez N, Vanhaeren H, Inzé D: Leaf size control: complex coordination of cell division and expansion. Trends Plant Sci 2012, 17(6):332-340.

9. Mizukami Y, Fischer RL: Plant organ size control: AINTEGUMENTA regulates growth and cell numbers during organogenesis. Proc Natl Acad Sci USA 2000, 97(2):942-947.
10. Donnelly PM, Bonetta D, Tsukaya H, Dengler RE, Dengler NG: Cell cycling and cell enlargement in developing leaves of Arabidopsis. Dev Biol 1999, 215(2):407-419.

11. Kazama T, Ichihashi Y, Murata S, Tsukaya H: The mechanism of cell cycle arrest front progression explained by a KLUH/CYP78A5-dependent mobile growth factor in developing leaves of Arabidopsis thaliana. Plant Cell Physiol 2010, 51(6):1046-1054.

12. Andriankaja M, Dhondt S, De Bodt S, Vanhaeren H, Coppens F, De Milde L, Muhlenbock P, Skirycz A, Gonzalez N, Beemster GT, Inzé D: Exit from proliferation during leaf development in Arabidopsis thaliana: a not-sogradual process. Dev Cell 2012, 22(1):64-78.

13. Lu D, Wang T, Persson S, Mueller-Roeber B, Schippers JH: Transcriptional control of ROS homeostasis by KUODA1 regulates cell expansion during leaf development. Nat Commun 2014, 5:3767.

14. Johnson K, Lenhard M: Genetic control of plant organ growth. New Phytol 2011, 191(2):319-333.

15. Hepworth J, Lenhard M: Regulation of plant lateral-organ growth by modulating cell number and size. Curr Opin Plant Biol 2014, 17:36-42.

16. Krizek BA: Ectopic expression AINTEGUMENTA in Arabidopsis plants results in increased growth of floral organs. Dev Genet 1999, 25(3):224-236.

17. Hu Y, Xie Q, Chua NH: The Arabidopsis auxin-inducible gene ARGOS controls lateral organ size. Plant Cell 2003, 15(9):1951-1961.

18. Anastasiou E, Kenz S, Gerstung M, MacLean D, Timmer J, Fleck C, Lenhard M: Control of plant organ size by KLUH/CYP78A5-dependent intercellular signaling. Dev Cell 2007, 13(6):843-856.

19. Disch S, Anastasiou E, Sharma VK, Laux T, Fletcher JC, Lenhard M: The E3 ubiquitin ligase BIG BROTHER controls Arabidopsis organ size in a dosage-dependent manner. Curr Biol 2006, 16(3):272-279.

20. Li Y, Zheng L, Corke F, Smith C, Bevan MW: Control of final seed and organ size by the DA1 gene family in Arabidopsis thaliana. Genes Dev 2008, 22(10):1331-1336.

21. Xu R, Li Y: Control of final organ size by Mediator complex subunit 25 in Arabidopsis thaliana. Development 2011, 138(20):4545-4554.

22. Cho HT, Cosgrove DJ: Altered expression of expansin modulates leaf growth and pedicel abscission in Arabidopsis thaliana. Proc Natl Acad Sci USA 2000, 97(17):9783-9788.

23. Hu Y, Poh HM, Chua NH: The Arabidopsis ARGOS-LIKE gene regulates cell expansion during organ growth. Plant J 2006, 47(1):1-9.

24. Deprost D, Yao L, Sormani R, Moreau M, Leterreux G, Nicolai M, Bedu M, Robaglia C, Meyer C: The Arabidopsis TOR kinase links plant growth, yield, stress resistance and mRNA translation. EMBO Rep 2007, 8(9):864-870.

25. Kurepa J, Wang S, Li Y, Zaitlin D, Pierce AJ, Smalle JA: Loss of $26 \mathrm{~S}$ proteasome function leads to increased cell size and decreased cell number in Arabidopsis shoot organs. Plant Physiol 2009, 150(1):178-189.

26. Sonoda Y, Sako K, Maki Y, Yamazaki N, Yamamoto H, Ikeda A, Yamaguchi J: Regulation of leaf organ size by the Arabidopsis RPT2a 19S proteasome subunit. Plant J 2009, 60(1):68-78.

27. Tsukaya $\mathrm{H}$ : Interpretation of mutants in leaf morphology: genetic evidence for a compensatory system in leaf morphogenesis that provides a new link between cell and organismal theories. Int Rev Cytol 2002, 217:1-39.

28. Beemster GT, Fiorani F, Inzé D: Cell cycle: the key to plant growth control? Trends Plant Sci 2003, 8(4):154-158.

29. Tsukaya $\mathrm{H}$ : Controlling size in multicellular organs: focus on the leaf. PLOS Biol 2008, 6(7):e174

30. Kawade K, Horiguchi G, Tsukaya H: Non-cell-autonomously coordinated organ size regulation in leaf development. Development 2010, 137(24):4221-4227.

31. Feng G, Qin Z, Yan J, Zhang X, Hu Y: Arabidopsis ORGAN SIZE RELATED1 regulates organ growth and final organ size in orchestration with $A R G O S$ and ARL. New Phytol 2011, 191(3):635-646.

32. Guo M, Rupe MA, Wei J, Winkler C, Goncalves-Butruille M, Weers BP, Cerwick SF, Dieter JA, Duncan KE, Howard RJ, Hou Z, Löffler CM, Cooper M, Simmons CR: Maize ARGOS1 (ZAR1) transgenic alleles increase hybrid maize yield. J Exp Bot 2014, 65(1):249-260.

33. Johnston GC, Pringle JR, Hartwell LH: Coordination of growth with cell division in the yeast Saccharomyces cerevisiae. Exp Cell Res 1977, 105(1):79-98. 
34. Neufeld TP, de la Cruz AFA, Johnston LA, Edgar BA: Coordination of growth and cell division in the Drosophila wing. Cell 1998, 93(7):1183-1193.

35. Melaragno JE, Mehrotra B, Coleman AW: Relationship between endopolyploidy and cell-size in epidermal tissue of Arabidopsis. Plant Cell 1993, 5(11):1661-1668.

36. Sugimoto-Shirasu K, Roberts K: "Big it up": endoreduplication and cell-size control in plants. Curr Opin Plant Biol 2003, 6(6):544-553.

37. Kalve $S$, De Vos D, Beemster GT: Leaf development: a cellular perspective. Front Plant Sci 2014, 5:362.

38. Jing Y, Cui D, Bao F, Hu Z, Qin Z, Hu Y: Tryptophan deficiency affects organ growth by retarding cell expansion in Arabidopsis. Plant J 2009, 57(3):511-521.

39. Nicholas KB, Nicholas HBJ: GeneDoc: A tool for editing and annotating multiple sequence alignments. EMBNEW NEWS 1997, 4:14.

40. Liu Y, Cui S, Wu F, Yan S, Lin X, Du X, Chong K, Schilling S, Theissen G, Meng Z: Functional conservation of MIKC*-Type MADS box genes in Arabidopsis and rice pollen maturation. Plant Cell 2013, 25(4):1288-1303.

41. Curtis MD, Grossniklaus U: A gateway cloning vector set for highthroughput functional analysis of genes in planta. Plant Physiol 2003, 133(2):462-469.

42. Qin G, Gu H, Zhao Y, Ma Z, Shi G, Yang Y, Pichersky E, Chen H, Liu M, Chen Z, Qu LJ: An indole-3-acetic acid carboxyl methyltransferase regulates Arabidopsis leaf development. Plant Cell 2005, 17(10):2693-2704.

43. Clough SJ, Bent AF: Floral dip: a simplified method for Agrobacteriummediated transformation of Arabidopsis thaliana. Plant J 1998, 16(6):735-743

44. Yang K, Wang H, Xue S, Qu X, Zou J, Le J: Requirement for A-type cyclindependent kinase and cyclins for the terminal division in the stomatal lineage of Arabidopsis. J Exp Bot 2014, 65(9):2449-2461.

45. Nelissen H, Rymen B, Coppens F, Dhondt S, Fiorani F, Beemster GT: Kinematic analysis of cell division in leaves of mono- and dicotyledonous species: a basis for understanding growth and developing refined molecular sampling strategies. Methods Mol Biol 2013, 959:247-264.

46. Galbraith DW, Harkins KR, Maddox JM, Ayres NM, Sharma DP, Firoozabady E: Rapid flow cytometric analysis of the cell-cycle in intact plant-tissues. Science 1983, 220(4601):1049-1051.

\section{Submit your next manuscript to BioMed Central and take full advantage of:}

- Convenient online submission

- Thorough peer review

- No space constraints or color figure charges

- Immediate publication on acceptance

- Inclusion in PubMed, CAS, Scopus and Google Scholar

- Research which is freely available for redistribution 